\title{
Studies of Electrical and Physico- Mechanical Properties of EPDM Structure Foams
}

\author{
A.M.Y. El-Lawindy \\ Department of physics, Faculty of Science, Suez Canal University, \\ Ismailia, Egypt
}

\begin{abstract}
Ethylene-propylene-diene terpolymer, EPDM structure foams of different apparent densities was compounded by using different concentration of foaming agent, azodicarboamide, ADC/K. The electrical conductivity was measured under different compression strains; 0\%, 10\%, 30\%, 40\%, 50\% and $60 \%$. The stress relaxation at different compressive stresses was also measured. The change of conductivity under the effect of compression strains was explained by the aid of calculated carbon black shape factor. The effect of the foam structure on the electrical properties ( $d c$ and $a c$ ) of the reinforced carbon black- EPDM system was studied.
\end{abstract}

\section{Introduction:}

Elastomer foams meet increasing technological applications. Its low density with reasonable strength characterizes reinforced structure rubber foams. This makes it a useful construction material, as well as pressure sensitive composites, scale. There is an enormous interest in manufacturing rubber foams, but the knowledge of the dependence of macrostructure on microstructure of these foams is limited.

On the other hand, electrical conductivity of polymers can be provided by incorporation of conductive materials such as carbon black. The introduction of carbon black allows them to envisage the elaboration of polymers possessing the conductive properties of carbon black and the varied properties of polymers.

amvlawindy@hotmail.com 
The present work is a continuation of a program to study the physical properties of structure foam. The rubber latex used in this study was nitrilebutadiene rubber, NBR, and ethylene-propylene-diene terpolymer, EPDM. This choice was based on their common use as general-purpose rubber, in addition to their distinguished property [1].

In previous work [2-4], structure foam rubber [2], with density as low as $420 \mathrm{~kg} / \mathrm{m}^{3}$ and $270 \mathrm{~kg} / \mathrm{m}^{3}$ for EPDM and NBR respectively, were obtained by an easy and simple technique affecting the mechanical properties of the unfoamed matrices only slightly. The analysis of the stress-strain data was based on the assumption that the foaming agent caused carbon dilution in the matrix. The decrease of carbon black volume fraction was found linearly dependent on the foam concentration. The calculated shape factor was found dependent on the foaming agent concentration. The analysis of the I-V characteristic [3] for these foams showed two distinct regions of conduction behavior: Ohmic up to $\sim 400$ volt and space charge limited conduction, SCLC, above 400 volt. The conductivity as a function of temperature was measured. There was a competition between two conduction mechanisms, tunneling and thermal activation. The inter-particle separation distances between carbon aggregates were calculated and were found increasing by increasing the foaming agent concentration. For NBR [4], the swelling factors in benzene and gasoline as a function of time were measured to elucidate the structure of these foams. That study was assisted by the I-V characteristics, which were measured under the effect of compressive strains. The measured conductivity showed deviation from Ohmicity below 400 volt. This effect was attributed to the change of carbon black structure as well as to the lateral deformation of gas cells. This assumption was confirmed by calculating the shape factor. The calculated free carrier mobility showed that the conduction mechanism belonged to the conventional band model.

The aim of the present study was to systematically investigate the effect of adding different concentrations of foaming agent, $\mathrm{ADC} / \mathrm{K}$ on the ac electrical properties as well as the physhico-mechanical properties of EPDM, in an attempt to improve these properties and also to study the stress relaxation at different compressive stresses.

\section{Experimental Technique:}

The foamed EPDM rubber composites were compounded according to the recipe shown in Table (1). A two-roll mill was used. The ingredients were added in the same order as listed. 
Table 1: Ingredients of the investigated foamed EPDM (E's) rubber composites. MBTS is dibenzthiazole disulphide, PBN is phenyl- $\beta$-naphthyl amine, and phr is part per hundred part of rubber by weight.

\begin{tabular}{|l|c|c|c|c|}
\hline $\begin{array}{c}\text { Sample name } \\
\text { Ingredients (phr) }\end{array}$ & E0 & E5 & E10 & E15 \\
\hline EPDM & $\mathbf{1 0 0}$ & $\mathbf{1 0 0}$ & $\mathbf{1 0 0}$ & $\mathbf{1 0 0}$ \\
\hline Stearic acid & 2 & 2 & 2 & 2 \\
\hline Zinc Oxide & 5 & 5 & 5 & 5 \\
\hline HAF-N330 & 50 & 50 & 50 & 50 \\
\hline Processing oil & 15 & 15 & 15 & 15 \\
\hline MBTS & 2 & 2 & 2 & 2 \\
\hline PBN & 1 & 1 & 1 & 1 \\
\hline Sulpher & 2.5 & 2.5 & 2.5 & 2.5 \\
\hline ADC/K & $\mathbf{0}$ & $\mathbf{5}$ & $\mathbf{1 0}$ & $\mathbf{1 5}$ \\
\hline
\end{tabular}

The specification of the used two-roll mill is as follow: length: $0.3 \mathrm{~m}$, radius: $0.15 \mathrm{~m}$, speed of slow roll: $18 \mathrm{rpm}$ and gear ratio: 1.4 . The compounded rubber was left for 24 hours before vulcanization.

The specimens were compression molded into cylinders of $\sim 1 \times 10^{-4} \mathrm{~m}^{2}$ surface and $\sim 0.01 \mathrm{~m}$ in height. The vulcanization was conducted under a heating press (KARL KOLB, Germany) at a pressure of $P=0.40 \mathrm{MPa}$. Many trials were conducted to obtain an optimum condition of temperature and time of vulcanization without samples being collapsed. The optimum conditions of temperature and time was $T=423 \mathrm{~K}$ and $t=30 \mathrm{~min}$. The vulcanized samples were shelf aged for 48 hours before testing. The mixing time and vulcanization conditions were fixed for all samples. Brass electrodes were attached to the parallel faces of samples during vulcanization. Material tester (AMETEK, USA) was facilitated in this experiment. A digital force gauge (Hunter Spring ACCU Force II, $0.01 \mathrm{~N}$ resolution, USA) of $50 \mathrm{~N}$ capacity was used to measure stress forces. In dc electrical measurement a digital electrometer (616 Kiethly, USA) was used. Permittivity $\left(\varepsilon^{\prime}\right)$ and dielectric loss $\left(\varepsilon^{\prime \prime}\right)$ for the denoted samples were measured at different frequencies ranging from $40 \mathrm{~Hz}$ to $5 \mathrm{MHz}$. An LCR meter (Hioki- Japan) bridge was used. The capacitance (C) and the loss tangent (tan $\delta$ ) were obtained directly from the bridge from which $\varepsilon$ ' and $\varepsilon$ " were calculated. The accuracy for $\varepsilon$ ' was $\sim \pm 1 \%$ and for $\varepsilon$ " was $\sim \pm 2 \%$. 


\section{Results and Discussion:}

\section{I. Electrical Properties:}

In an attempt to obtain foam rubber with improved electrical as well as mechanical properties, azodicarbonamide were formulated together with carbon black reinforced EPDM in different concentrations (Table 1). The relative permittivity $\left(\varepsilon^{\prime}\right)$ and dielectric loss $\left(\varepsilon^{\prime \prime}\right)$ for the prepared samples (E0, E5, E10, E15) were studied over the frequency range from $40 \mathrm{~Hz}$ to $5 \mathrm{MHz}$ at room temperature ( 300 K).

It is evident from Fig. (1), which represents the variation of $\varepsilon$ with frequency, that the value of $\varepsilon$ decreases with increasing frequency. The decrease of $\varepsilon$ with frequency shows an anomalous dispersion. Moreover, $\varepsilon$ decreases rapidly with the addition of azodicarbonamide. This could be attributed to the effect of azodicarbonamide produced cells which disrupt the chains of contacting particles and decreases the dielectric permittivity so rapidly.

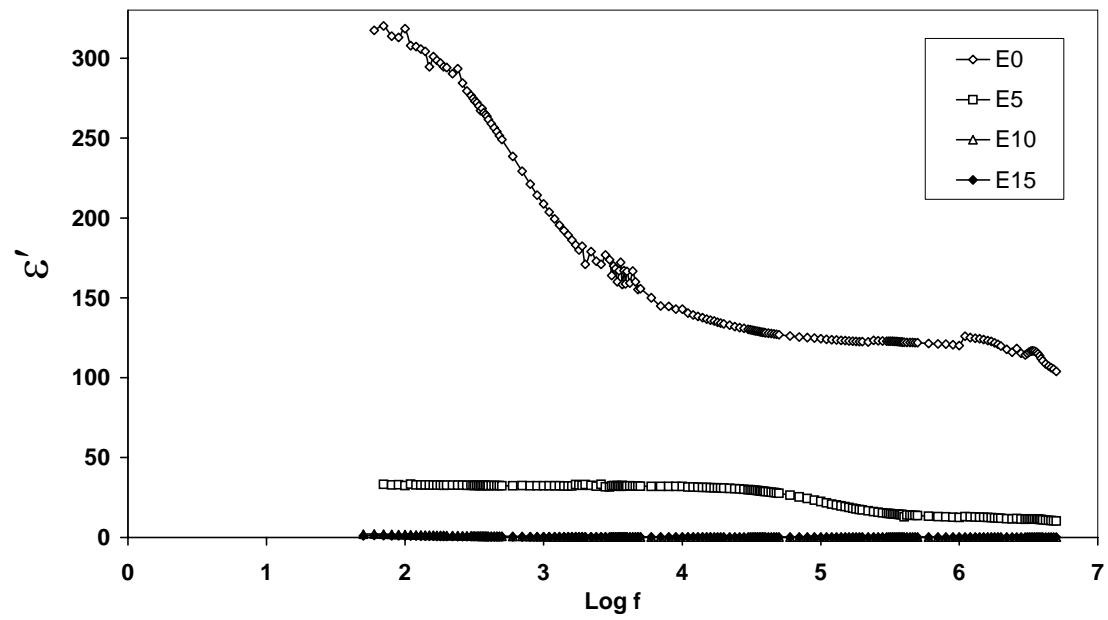

Fig (1): The variation of the relative permittivity, $\varepsilon$, as a function frequency in $\mathrm{Hz}$, for all samples.

In Figure (2) the dielectric losses, $\varepsilon^{\prime \prime}$, at $\sim 300{ }^{\circ} \mathrm{K}$ as a function of frequency are shown for unloaded and loaded EPDM samples with ADC/K content. Unloaded EPDM sample and loaded specimens with $5 \mathrm{phr}$ of ADC/K show peak losses that shift to higher frequency for foamed sample which contains less carbon black. It is known that filled specimens with carbon black always show greater losses than the unfilled one in the approximate whole frequency range. Concerning dielectric losses, $\varepsilon$ ", as a function of frequency, 
Fig. (2), data in tested frequency range (for 0 and $5 \mathrm{phr} A D C / K$ ) depict a relaxation process that may be attributed to an interfacial polarization known as Maxwell-Wagner-Sillars (MWS) effect [5]. This phenomenon appears in heterogeneous media consisting of phases with different dielectric permittivity and conductivities and is due to accumulation of charges at interfaces. Analysis of the process [6] shows a Debye-type relaxation and the frequency dependencies of the permittivity components of the composite systems are expressed by:

$$
\begin{aligned}
& \varepsilon^{\prime}(\omega)=\varepsilon_{\infty}+\frac{\varepsilon_{o}-\varepsilon_{\infty}}{1+\omega^{2} \tau^{2}} \\
& \varepsilon^{\prime \prime}(\omega)=\frac{\left(\varepsilon_{o}-\varepsilon_{\infty}\right) \omega \tau}{1+\omega^{2} \tau^{2}}+\frac{\sigma}{\omega}
\end{aligned}
$$

where $\varepsilon_{0}$ and $\varepsilon_{\mathrm{oo}}$ are the static and dynamic permittivity of the system , $\tau$ is the relaxation time, and $\sigma$ is the ac conductivity.

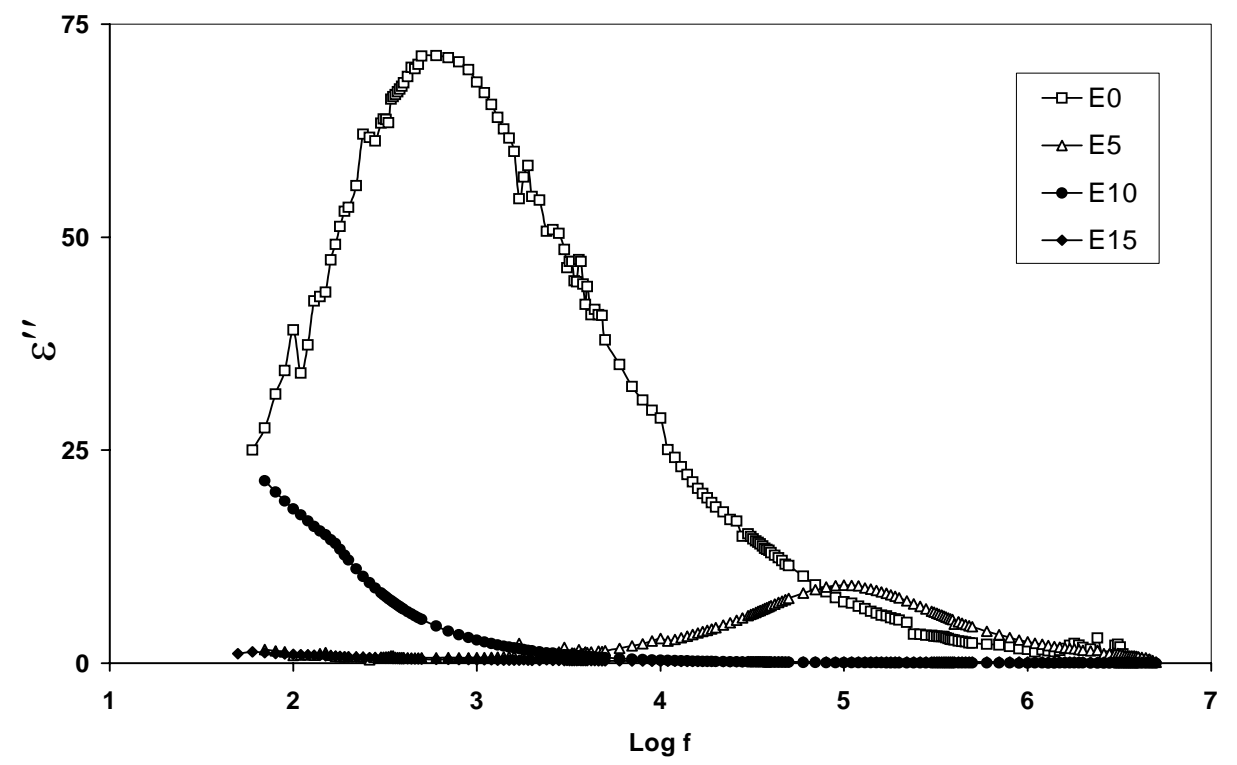

Fig .(2): The variation of the dielectric loss, $\varepsilon "$, as a function frequency in $\mathrm{Hz}$, for all samples.

In our case, heterogeneity in the unfoamed sample is due to HAF black particles and polymer (EPDM). In the foamed specimens, there exists one more interface between gas cells and polymer. Increase in foam contents ( $5 \mathrm{phr}$ ) shifts the relaxation peak to higher frequency. Heterogeneity is greater in foamed 
specimens and the MWS effects arising from the carbon-polymer interface is superimposed on that of the polymer-gas cells. As frequency is increased, $\varepsilon$ decreases quickly according to equation (2) because the term $\frac{\sigma}{\omega}$ becomes insignificant. Values of $\varepsilon$ and $\varepsilon$ " are calculated from Equations (1) and (2) and fitted well with the experimental data for samples E0 and E5 as shown in Fig. (3).
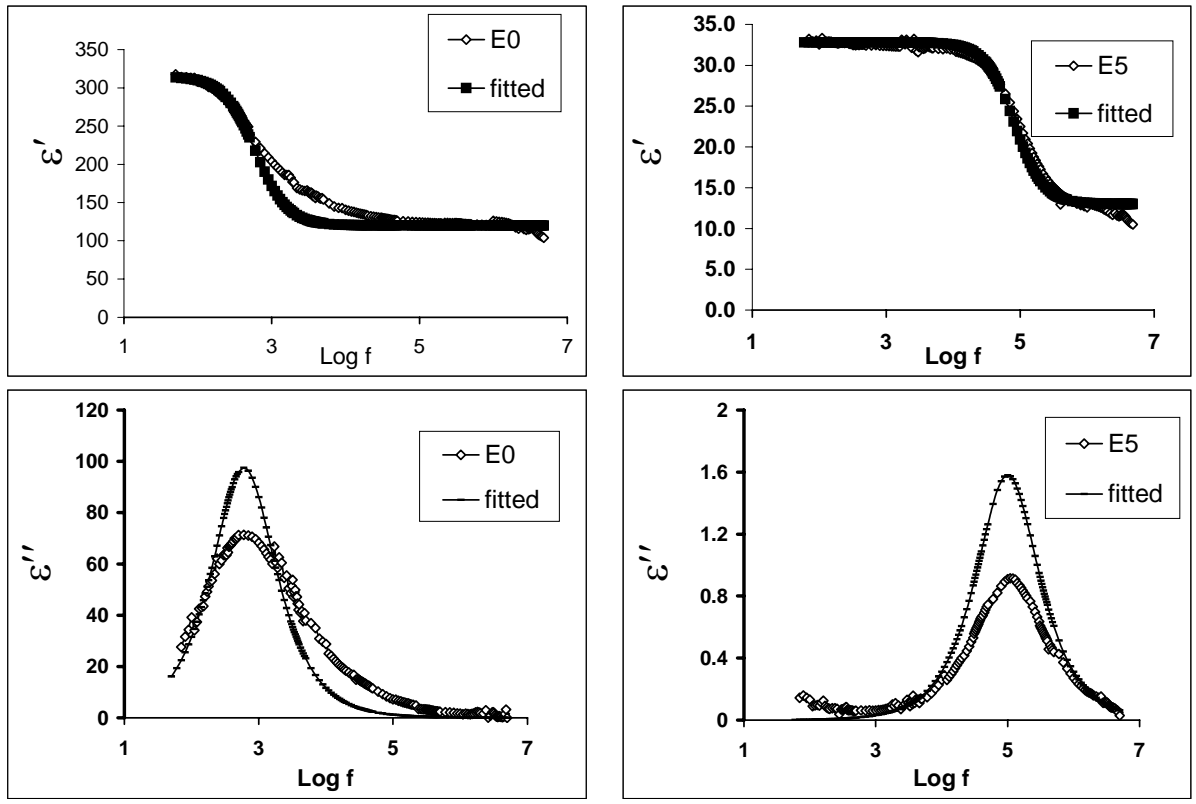

Fig. (3): The fitted curves calculated from equation (1) and (2) compared to measured values of $\varepsilon$ and $\varepsilon$.

\section{3-2. Physico- mechanical Measurement:}

The dilution of carbon black owing to the addition of ADC/K foam agent as detected by the ac measurement leads to the increasing behavior in the interspacing distance between carbon particles. This could be confirmed by the $\mathrm{I}-\mathrm{V}$ measurements under compressive strains.

The I-V characteristics at different compressive strain were measured for all samples, Fig (4). The data showed no remarkable deviation from ohmicity, as was the case in NBR [3]. The conductivity as a function of compressive strain is illustrated in Fig (5). It is seen that the conductivity is steeply decreasing with increasing compression strains. This reflects either the increase of inter-particle distances or the break up of carbon black structure. 

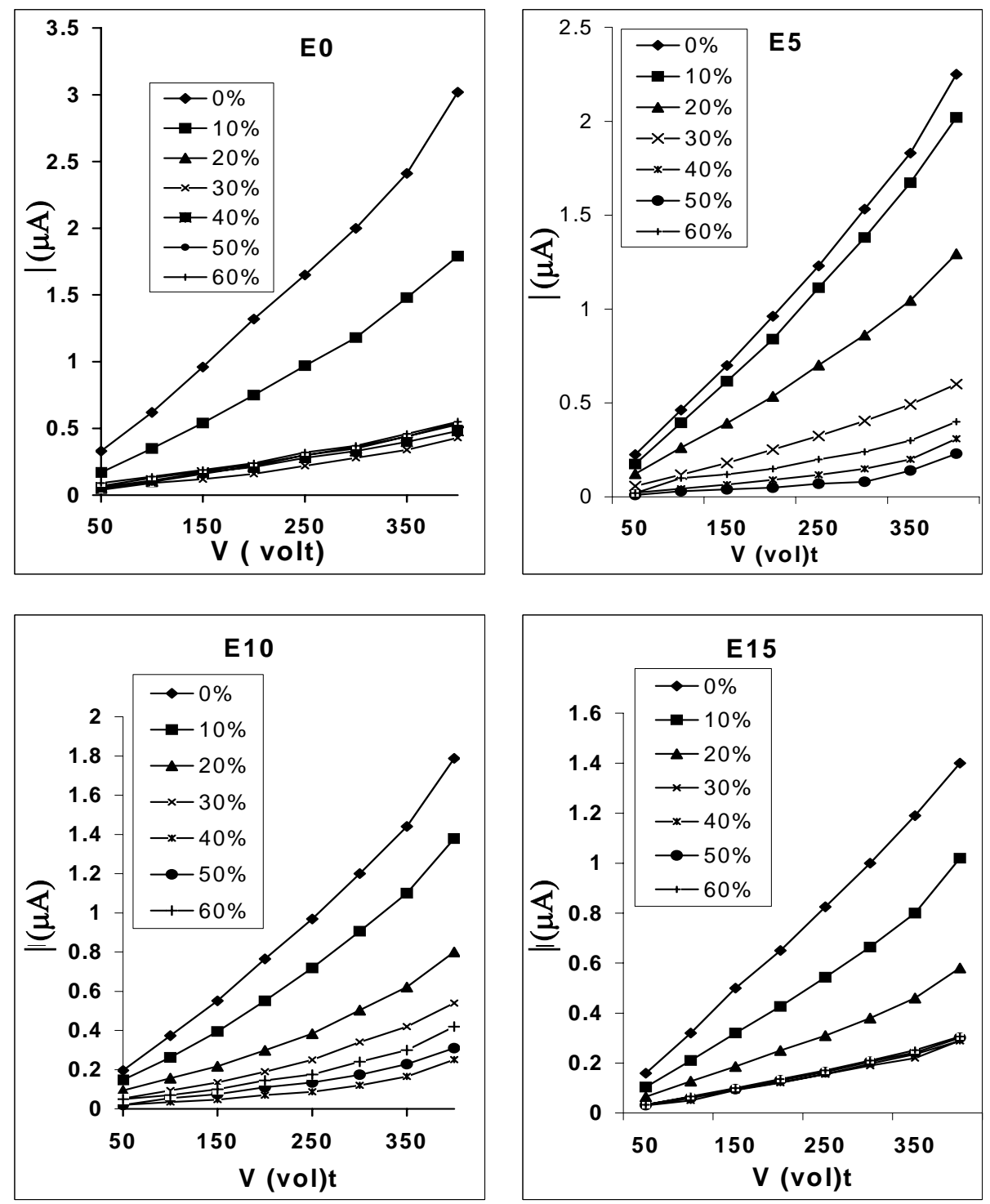

Fig. (4): I-V characteristics for all samples at different compression strain ratios, $0 \%, 10 \%, 20 \%, 30 \%, 40 \%, 50 \%$ and $60 \%$.

This assumption may be confirmed by calculating the shape factor of carbon filler by using a modified Guth [7] equation:

$$
\sigma_{\mathrm{s}} / \sigma_{\mathrm{o}}=1-\mathrm{f} \phi_{f}-\mathrm{f}^{2} \phi_{\mathrm{f}}^{2}
$$


where $\sigma_{\mathrm{s}}$ is the conductivity under strain, $\sigma_{0}$ is the conductivity under no strain, $\mathrm{f}$ is the shape factor, and $\phi_{f}$ is the volume fraction of carbon filler. The positive signs in Guth equation are replaced by negative signs to account for the decrease of conductivity due the increase of foaming agent concentration. Figure (6) depicts the calculated f-values as a function of compressive strains for EPDM foams. It is noticed that the shape factor is markedly increasing, which means that the shape is getting more prolate, i.e. may take the shape of thin tubes of small diameter relative to its length, and are arranged in planes perpendicular to the direction of compression. In addition, the gas cells are inflated in lateral directions reducing the probability of interconnection between these newly formed aggregates. Consequently the conductivity is decreasing.

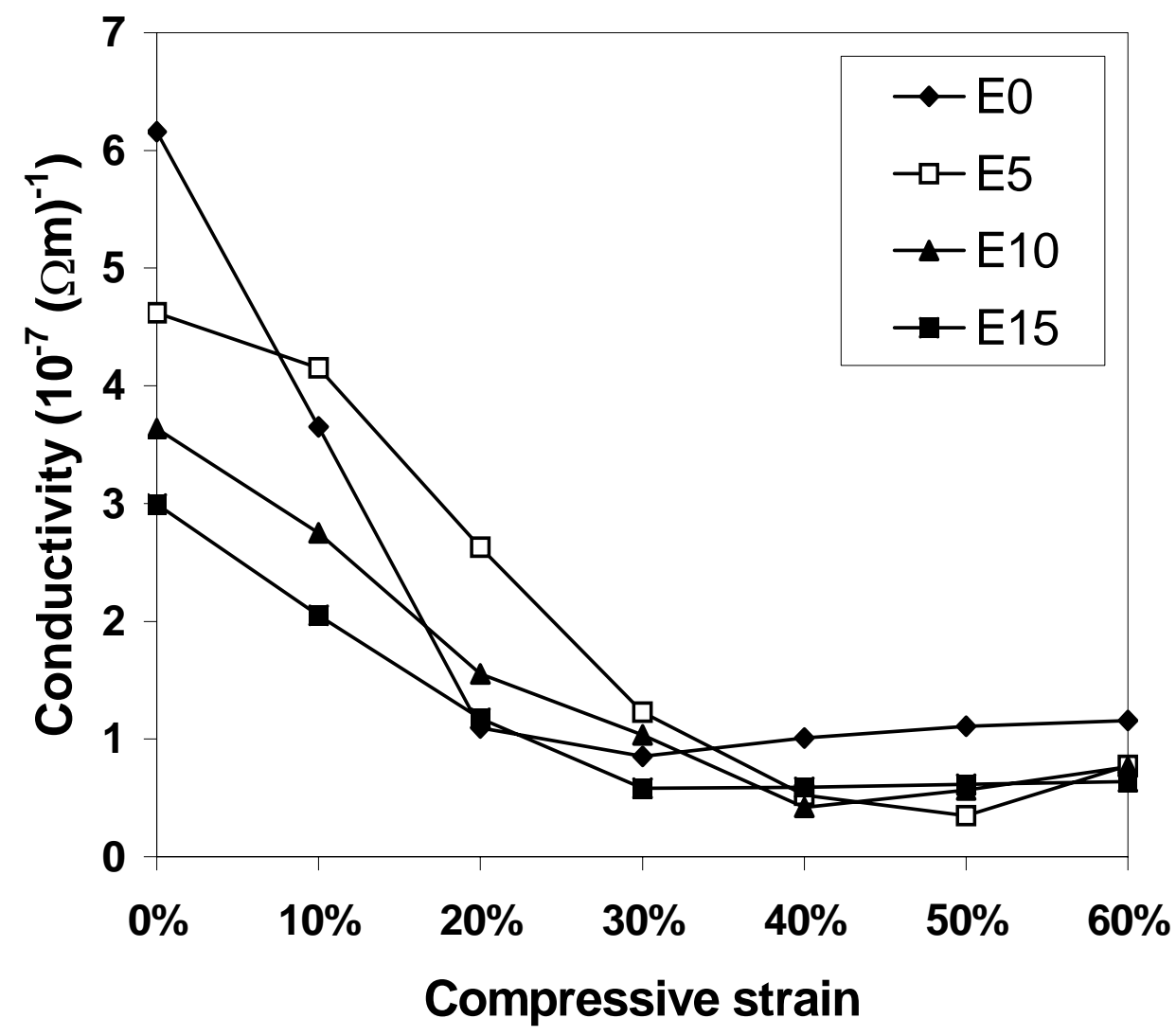

Fig. (5): Measured dc conductivity, $\sigma(\Omega \mathrm{m})^{-1}$ at different compressive strain ratios, $0 \%, 10 \%, 20 \%, 30 \%, 40 \%, 50 \%$ and $60 \%$. 


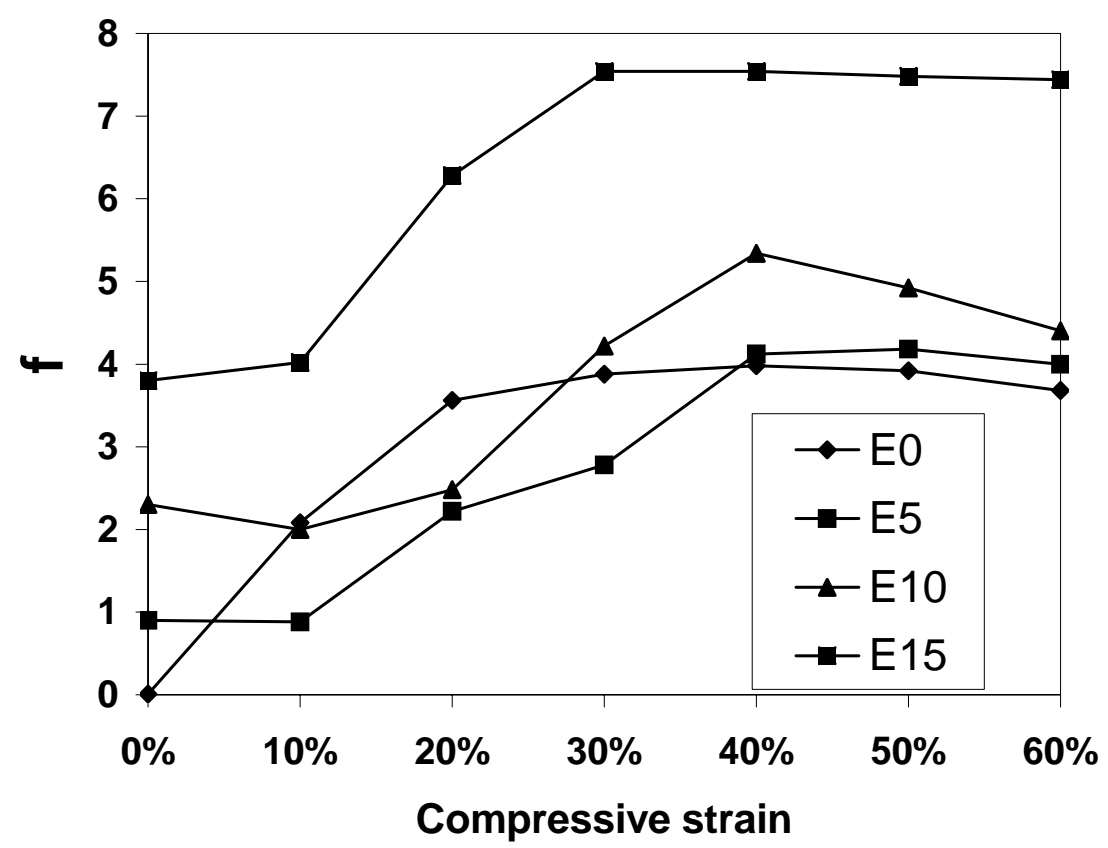

Fig. (6): Calculated shape factors at different compressive stain ratios, $0 \%$, $10 \%, 20 \%$, 30\%, 40\%, 50\% and 60\%.

In order to calculate the active volumes of these aggregates, the conductivity relaxation were measured at different compressions. Following the calculation of Thompson et al [8], a proposed relation between pressure and conductivity, $\sigma$, is as follows:

$$
\sigma=g(t, T, \phi) \exp (\mathrm{Vp} / \mathrm{KT})
$$

Where $\mathrm{g}$ is a function of time, t, the temperature, $\mathrm{T}$, and the structure, $\phi . \mathrm{V}$ is the activation volume, the physical meaning of which is associated with the region of the space where conduction events take place, $\mathrm{K}$ is Boltzman's constant. When a pressure step is made over the sample at constant temperature and structure, the change in the derivative of conductivity, $\dot{\sigma}=\frac{d \sigma}{d t}$, can be measured, then one has;

$$
V=-\frac{K T}{p} \frac{\ln \dot{\sigma}_{2} / \ln \dot{\sigma}_{1}}{\ln p_{2} / p_{1}}
$$


The negative sign takes into consideration the decrease of conductivity with increasing pressure. Figure (7) shows the behavior of step pressure conductivity as a function of time at different compressions, 0.05, 0.15, and 0.25 Mpa respectively. The calculated activation volumes in addition to the previously [3] calculated inter-particle distances, D, are listed in Table (2).
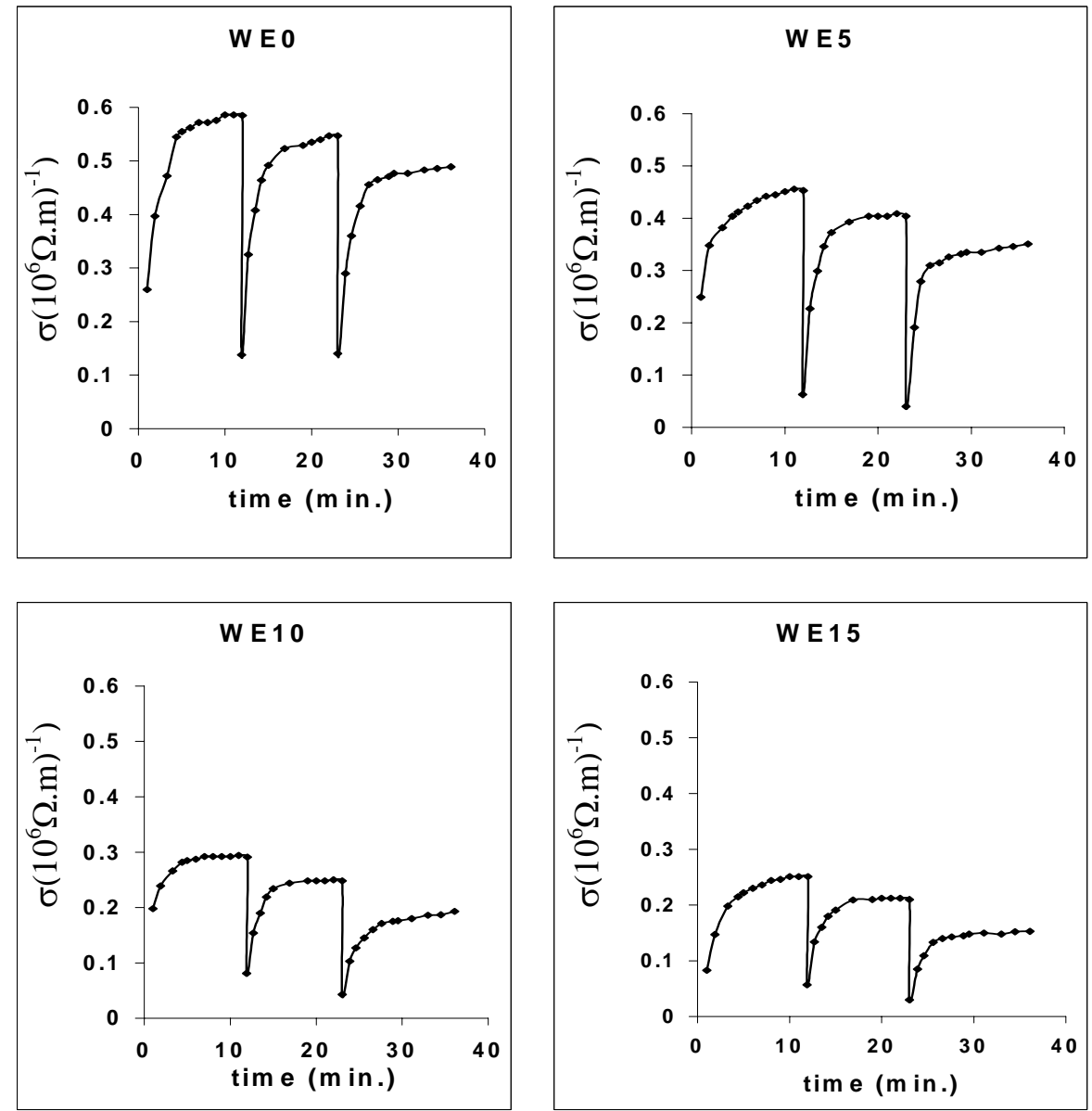

Fig. (7): Conductivity relaxation at step pressure, $0.05 \mathrm{MPa}, 0.15 \mathrm{MPa}$, and $0.25 \mathrm{MPa}$, respectively.

Table (2): The calculated active volumes, $\mathrm{V}$, and inter-particle distances, D, for all samples.

\begin{tabular}{|c|c|c|c|c|}
\hline Sample & E0 & E5 & E10 & E15 \\
\hline $\mathrm{V}\left(\mathrm{nm}^{3}\right)$ & $21.0 \pm 3.1$ & $39.6 \pm 7.0$ & $33.2+4.2$ & $50.7 \pm 8.2$ \\
\hline $\mathrm{D}(\mathrm{nm})$ & $481 \pm 11$ & $513 \pm 13$ & $596+12$ & $612 \pm 11$ \\
\hline
\end{tabular}


It is seen that increasing foam content increases the active volume and the inter-particle separation distances. Consequently, the conductivity at such low volume fraction of carbon black may be attributed to the increased mobility of rubber chain molecules.

\section{Conclusion:}

The results show the expected decrease in dielectric permittivity with increasing frequency. It also shows a decrease in dielectric permittivity with increasing foam content, as a result of carbon black dilution. These effects are also shown for dielectric loss. For low foam concentration, zero and five phr, the samples show loss peaks at $700 \mathrm{~Hz}$ and $109 \mathrm{kHz}$ respectively, and fitted well with Debye equations. For higher foam concentration, the specimens loose their dielectric properties impacted by carbon black, and its conductivity is mainly due to the increased mobility of rubber chain molecules. This is manifested by the increase of the active volumes with increasing foam content.

The measured I-V characteristics showed no remarkable deviation from Ohmicity upon compressive deformation of samples. The conductivity shows a dramatic decrease as the applied deformation increases. These changes may be attributed to the change of carbon black structure as well as the lateral deformation of gas cells. This assumption was confirmed by calculating the shape factor of carbon black filler.

\section{References:}

1. Whelan T., "Polymer Technology Dictionary", Chapman \& Hall, London, 1994

2. El-Lawindy A.M.Y., Abdel-Kader K.M., Mahmoud W.E. and Hassan; H.H. Polym. Int.; 51,(7), 601 (2002)

3. El-Lawindy A.M.Y., Mahmoud W.E.and Hassan H.H.; Egypt. J. Sol.,; 26, (1), 43 (2003)

4. El-Lawindy A.M.Y., Egypt. J. Sol., 25, (2), 295 (2002)

5. Blythe A.R., "Electrical Properties of Polymers", Cambridge Univ. Press, 1977, p. 65

6. Van Beek L.K.H., "Progress in Dielectrics" ed. J.B.Birks and J. Hart Hewood, london, 1967 7, p.69.

7. L.E.Robert and F.Landel, "Mechanical Properties of Polymer and Composites”, Marcel Dekker press, New York 1994, p 384

8. Thompson G.M., Besuden T.W., and. Beumel L.L, Rubb. Chem. and Tech. 64, 501 (1991). 